\title{
The observation of gamma-rays via neutral current interaction at Super-Kamiokande using the T2K neutrino beam
}

\author{
Yusuke Koshio*i \\ Okayama University \\ E-mail: koshio@okayama-u.ac.jp
}

\begin{abstract}
We report the first measurement of the neutral current quasi-elastic (NCQE) cross section on oxygen in neutrino-oxygen nucleus interactions using the T2K neutrino beam. This process produced nuclear deexcitation gamma-rays that were observed in the Super-Kamiokande water Cherenkov detector. We select event candidates using the T2K beam timing, and observed 43 event candidates in the $4-30 \mathrm{MeV}$ reconstructed energy region which is slightly below the theoretical prediction of 51.0 events. We observed an NCQE cross section of $1.55 \times 10^{-38} \mathrm{~cm}^{2}$ with a $68 \%$ confidence interval of $(1.22,2.20) \times 10^{-38} \mathrm{~cm}^{2}$.
\end{abstract}

16th International Workshop on Neutrino Factories and Future Neutrino Beam Facilities - NUFACT2014, 25 -30 August, 2014

University of Glasgow, United Kingdom

* Speaker.

${ }^{\dagger}$ for $\mathrm{T} 2 \mathrm{~K}$ collaboration 


\section{Introduction}

We present a measurement of nuclear deexcitation gamma-rays produced via neutrino-nucleus neutral current (NC) interactions in Super-Kamiokande (SK) using the T2K neutrino beam. In typical $\mathrm{NC}$ interactions, the final state of the neutrino and associated nucleon are not observed in a water Cherenkov detector; however, this process is observable since an electromagnetic shower from the deexcitation gamma-rays generates detectable Cherenkov photons. The original motivation of this study was to determine the number of neutrino events via this process from nearby supernovae in a water Cherenkov detector [1]. In the $20-30 \mathrm{MeV}$ region, which is a typical energy of supernova neutrinos, the inelastic scattering; $v+{ }^{16} \mathrm{O} \rightarrow v+{ }^{16} \mathrm{O}^{*}$ is the dominant source of gamma-ray production on oxygen. Other gamma-ray production is from the neutral current quasielastic (NCQE) interaction with nucleon knocked out; $v+{ }^{16} \mathrm{O} \rightarrow v+p+{ }^{15} \mathrm{~N}^{*}\left(v+n+{ }^{15} \mathrm{O}^{*}\right)$ [2]. It becomes dominant at several hundreds of $\mathrm{MeV}$ neutrino energy. For example, the cross section is one order of magnitude larger than the NC inelastic scattering at $500 \mathrm{MeV}$. This paper presents the first observation of neutrino-oxygen NCQE cross section using the T2K neutrino beam. There are two motivations for this measurement: 1) the NCQE interaction due to atmospheric neutrinos is one of the main backgrounds in astrophysical neutrinos within the $10 \mathrm{MeV}$ range, such as supernova relic neutrinos in SK [3, 4], and a direct measurement of this process with a known neutrino source will be useful for ongoing and proposed projects; and 2) NC samples can be used to search for sterile neutrino oscillation and low mass dark matter.

In a water Cherenkov detector, when this interaction happens, two signals are observed. One is the 'primary' gamma-rays produced by neutrino-nucleus interactions, which only occurs when a knocked out nucleon comes from the $1 p_{3 / 2}$ or $1 s_{1 / 2}$ state. The other is the 'secondary' gamma-rays produced when knocked out nucleons interact with other nuclei in the water causing additional deexcitations. Both types of gamma-rays have an energy of about $10 \mathrm{MeV}$ which can be detected in SK. The narrow band neutrino beam at J-PARC is used as the known neutrino source.

\section{T2K and Super-Kamiokande experiment}

The Tokai-to-Kamioka (T2K) experiment is a long-baseline neutrino oscillation experiment consisting of a neutrino beam, several near detectors, and Super-Kamiokande as a far detector. It is designed to determine the neutrino oscillation parameters, mixing angles $\theta_{13}$ and $\theta_{23}$, the mass hierarchy of $\Delta m_{32}^{2}$, and the CP violation phase. T2K has obtained the first evidence of $v_{\mu} \rightarrow$ $v_{e}$ appearance signal and determined a non-zero $\theta_{13}$ [5]. The off-axis neutrino beam, where the original proton beam is directed $2.5^{\circ}$ away from SK, is used in T2K because of the first maximum in the neutrino oscillation probability at the location of the far detector. The neutrino beam has a narrow peak with median energy $630 \mathrm{MeV}$ at SK. This narrow energy peak is also useful for the observation of this NCQE interaction cross section at a particular energy. It is not possible to measure energy dependence of this cross section because the invisible outgoing neutrino makes accurate energy reconstruction impossible.

The SK detector is a cylindrical tank (39.3 $\mathrm{m}$ in diameter and $41.4 \mathrm{~m}$ in height) filled with 50 kilotonnes of pure water and lined with 11,129 20 inch PMTs. It is located $1000 \mathrm{~m}$ underground (2,700 m water equivalent) in the Kamioka mine in Gifu Prefecture, Japan. The signal detection 
method in SK is that Cherenkov light generated in water from charged particles are observed by the PMTs [6]. SK started in 1996, and there are four experimental phases. The T2K experiment is the latest phase (SK-IV), which started in 2008 after new electronics (i.e., QBEE [7]) were installed. The energy of gamma-rays is similar to the recoil electron energy from solar neutrinos. The SK detector performance is well calibrated by several methods [8]. In the energy region of our measurement, the energy was measured at $0.5 \%$ precision by LINAC and DT calibrations $[9,10]$. The vertex resolution is $55 \mathrm{~cm}$, the angular resolution is $23^{\circ}$, and the energy resolution is $14 \%$ for an electron with $10 \mathrm{MeV}$.

There are two kinds of backgrounds for gamma-rays produced by the NCQE interaction: neutrino beam-related, (i.e., NC other interactions (NC non-QE) and charged current (CC) interactions), and neutrino beam-unrelated such as gamma-rays from radioactive impurities, decay electrons from stopping muons in the water, etc.

\section{Event simulation}

There are three steps for event simulation of this analysis. First, the neutrino flux from the beamline is simulated with FLUKA which is used for hadron production in the target, and Geant3 which is used for propagating hadronic interactions through the magnetic horns, target hall, decay volume, and beam dump. The second step is neutrino interaction simulations using the NEUT event generator. The NCQE cross section on oxygen is simulated using a spectral function model [2]. In order to predict deexcitation gamma-ray emission, an identification of the nucleus state after the neutrino interaction is needed. In the simple nucleus model, the nucleons in oxygen occupy three states: $1 p_{1 / 2}, 1 p_{3 / 2}$, and $1 s_{1 / 2}$. Since the $1 p_{1 / 2}$ hole is already the ground state, it produces no primary gamma-rays. In the case of knockout from $1 p_{3 / 2}$, the released energy of gamma-ray is 6.32 or $6.18 \mathrm{MeV}$ for a proton or a neutron, respectively. A $9.93 \mathrm{MeV}$ gamma-ray is also released from the $1 p_{3 / 2}$ proton hole. The $1 s_{1 / 2}$ hole state has several channels, usually including additional nucleon emission because of the large binding energy. The calculation of the branching ratio is based on the experiment that measured ${ }^{16} \mathrm{O}(\mathrm{p}, 2 \mathrm{p}){ }^{15} \mathrm{~N}$ [11] . The third step is that all the particles leaving the nucleus are simulated through the SK detector. The GCALOR which is installed in Geant 3 is used for the simulation of nucleons with nuclei in the water, for example, the secondary gamma-ray production. The transportation of all the particles in water, generating Cherenkov photons, and the response of the PMT and electronics are also simulated in this step.

\section{Analysis}

T2K RUN1-3 data from a neutrino exposure based on $3.01 \times 10^{20}$ protons on target were analyzed in this paper. Only events within \pm 100 nanosecond from the bunch were used to reduce beam-unrelated background. The deexcitation gamma-ray energy is in a range of several $\mathrm{MeV}$ up to $30 \mathrm{MeV}$ which is similar to the region for solar neutrinos, therefore, the event reconstruction (i.e., vertex, direction and energy) is the same as solar neutrino analysis in SK.

In order to remove radioactive impurities in the PMT glass and cover, and from rock around SK, the following cuts were applied: a standard fiducial volume cut of $2 \mathrm{~m}$ from the detector wall, a tight fiducial volume cut which depends on energy because of the nonuniform distribution of 


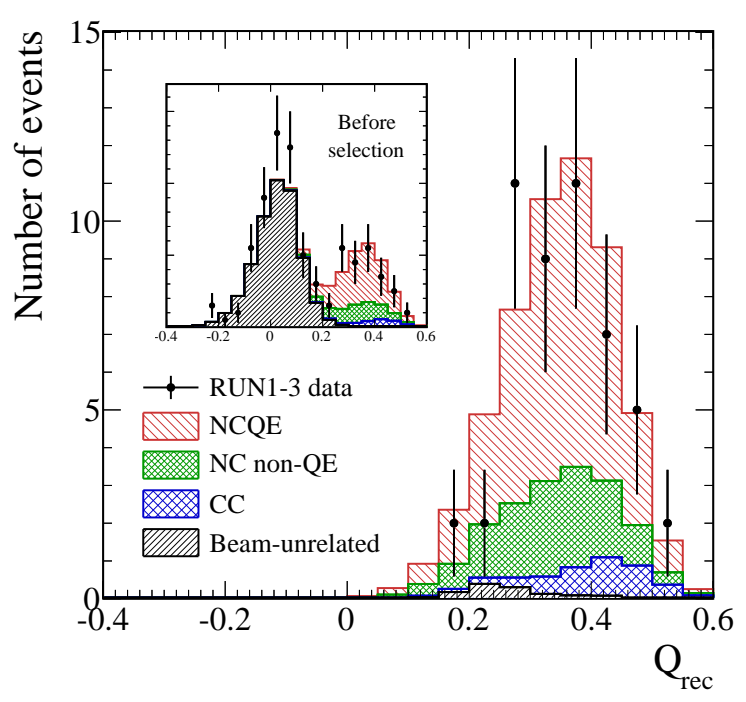

Figure 1: Distribution of the reconstruction fit quality value, $Q_{\text {rec }}$. The inset shows the distribution before the energy dependent cut on $Q_{r e c}$, but including the timing and fiducial volume cuts. The data are represented by points with statistical error bars and the expectation is represented by stacked histograms showing the NCQE signal and the NC non-QE, CC, and beam-unrelated background components.

the lower energy radioactive backgrounds, and a cut on the distance to the wall in the direction of the particle's momentum [12]. Even after those cuts, some of beam-unrelated background remain because of the misreconstruction; therefore, the fit quality value defined as $Q_{\text {rec }}=g_{v}^{2}-g_{d}^{2}$ was used, where $g_{v}$ is the goodness of the vertex reconstruction and $g_{d}$ is the direction goodness. This value was found to be effective to remove the background in solar neutrino analysis [12]. The $Q_{r e c}$ distribution is shown in Figure 1. The cut depends on energy and varies from 0.25 at its tightest at the low end of the energy to no cut above $11 \mathrm{MeV}$.

Finally, in order to reject the beam-related charged current interaction events, the following two cuts were applied. The first is the pre-activity cut, which uses timing from the previous event to remove decay electrons created from stopping muons. The second is the small Cherenkov opening angle cut to remove low energy muons.

\section{Results}

Figure 2 shows the observed event timing distribution in the final sample. Six events were found outside the bunch timing windows, which is consistent with the expected 3.6 random beam events. After all selections, 43 candidates were found in the $4-30 \mathrm{MeV}$ energy range, while 51.0 events were predicted by simulation. Among these predicted events, 34.80 events were expected to be NCQE signal,12.99 as NC non-QE, 2.04 as CC, and 1.20 as remaining beam-unrelated background.

Figure 3 shows the vertex distribution. No non-uniformities nor any biases with respect to the neutrino beam direction were found. Figure 4 shows the energy distribution. The observed energy 


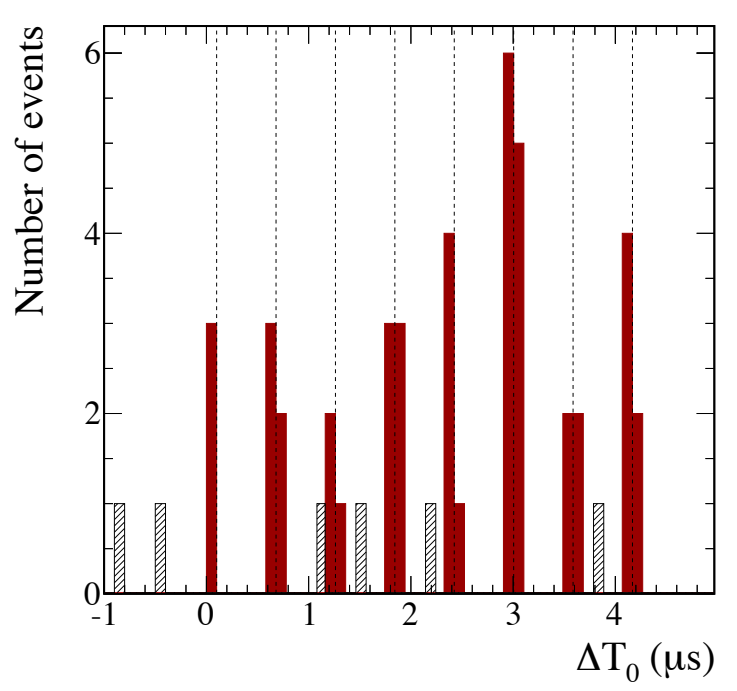

Figure 2: Timing from the beam trigger time distribution of the data sample, compared to the bunch center positions determined from high energy $\mathrm{T} 2 \mathrm{~K}$ neutrino events, indicated by eight dashed vertical lines. The on-timing and off-timing events are shown in solid and hashed, respectively.
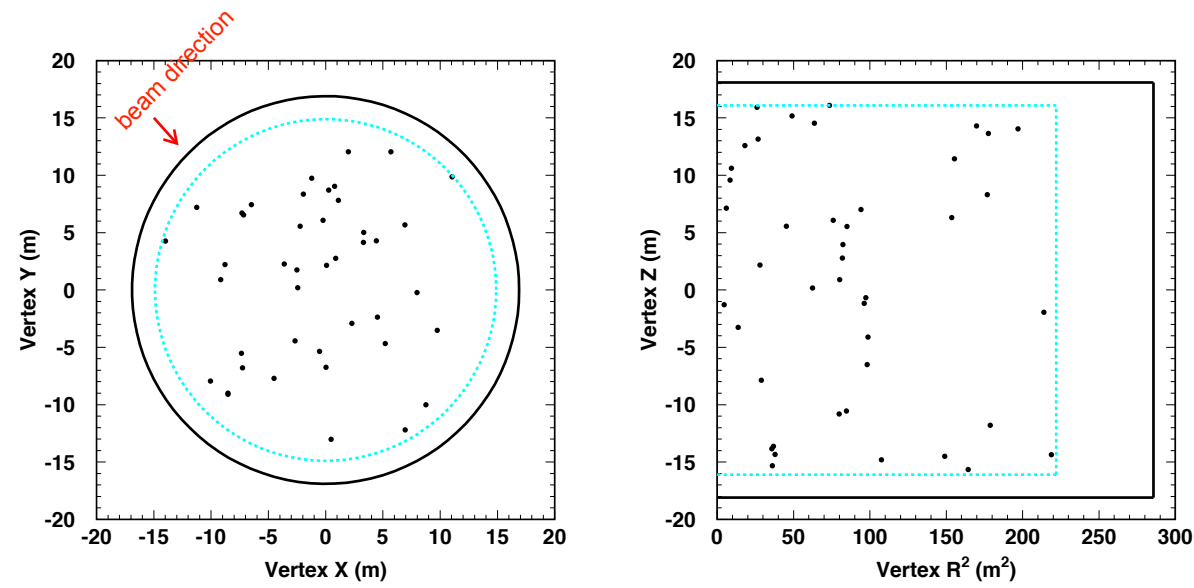

Figure 3: Vertex distribution of the final data sample. The solid and dotted lines indicate the boundaries of the inner detector and fiducial volume, respectively.

distribution had good agreement with the expectation with a peak due to $6 \mathrm{MeV}$ primary deexcitation gamma-rays. The high energy tail originated primarily from the contribution of additional secondary gamma-rays.

For this analysis, the Cherenkov angle of an event was calculated to compare with the expected hit pattern. It was calculated for every combination of three PMTs with hits, assuming the reconstructed vertex as the origin. For a single gamma-ray or electron, this peak will be close to the typical opening angle which is about $42^{\circ}$ in water, while the more isotropic light distributions from 


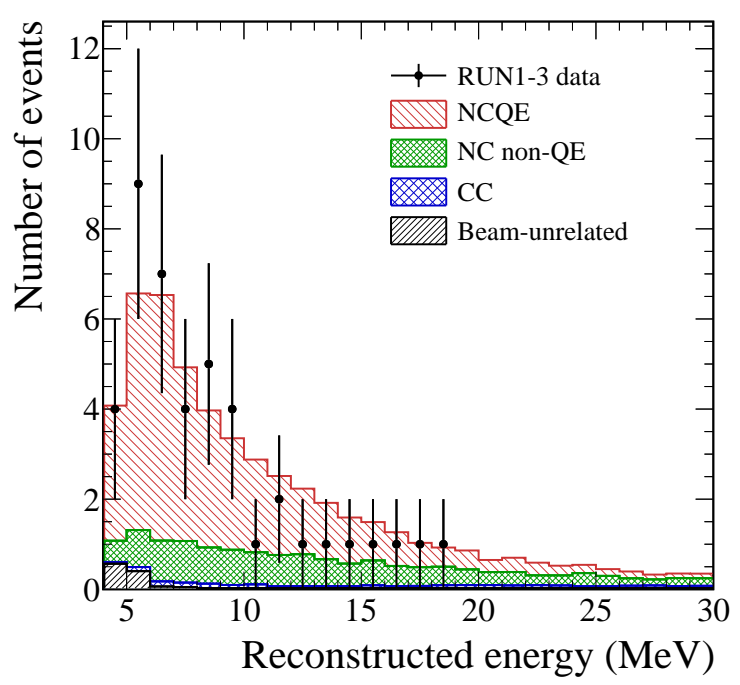

Figure 4: Reconstructed energy distribution for the final data sample and the MC expectation.

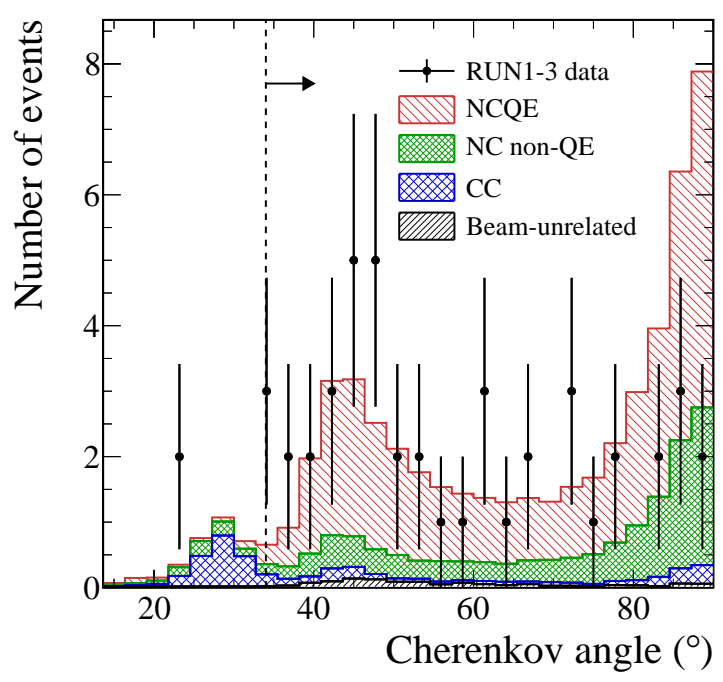

Figure 5: Cherenkov angle distribution in data and MC expectation. The expectation has a three-peak structure corresponding to low-momentum muons around $28^{\circ}$, single gamma-rays around $42^{\circ}$, and multiple gamma-rays around $90^{\circ}$. 
multiple particles will have peaks close to $90^{\circ}$. Figure 5 shows the Cherenkov angle distribution. In the region around $42^{\circ}$ data and expectation are consistent, while there is disagreement in the multiple particles peak region. The study of this discrepancy is still ongoing, but it is likely due to the approximations made in the model of secondary gamma-ray emission induced by the knocked out neutrons.

Finally, the NCQE cross section was calculated as follows;

$$
<\sigma_{v, N C Q E}^{o b s}>=\frac{N^{o b s}-N_{b k g}^{e x p}}{N^{e x p}-N_{b k g}^{e x p}}<\sigma_{v, N C Q E}^{\text {theory }}>
$$

where $<\sigma_{v, N C Q E}^{o b s}>$ is the observed flux-averaged NCQE cross section and $<\sigma_{v, N C Q E}^{\text {theory }}>=2.01 \times$ $10^{-38} \mathrm{~cm}^{2}$ is the flux-averaged cross section of the recent theoretical calculation [2]. The obtained flux-averaged neutrino-oxygen NCQE cross section was measured to be $1.55 \times 10^{-38} \mathrm{~cm}^{2}$ at a median neutrino flux energy of $630 \mathrm{MeV}$. The $68 \%$ confidence interval on the cross section was $(1.22,2.20) \times 10^{-38} \mathrm{~cm}^{2}$ including both statistical and systematic errors. The systematic errors calculated for each component were: $23 \%$ for NCQE signal, $25 \%$ for NC non-QE, $31 \%$ for CC, and $0.8 \%$ for remaining beam-unrelated background. The main contribution to the systematic errors comes from the production of secondary gamma-rays. Figure 6 shows the comparison of the NCQE cross section between data and expectation. The measurement was consistent with the recent theoretical calculation at the $68 \%$ confidence level.

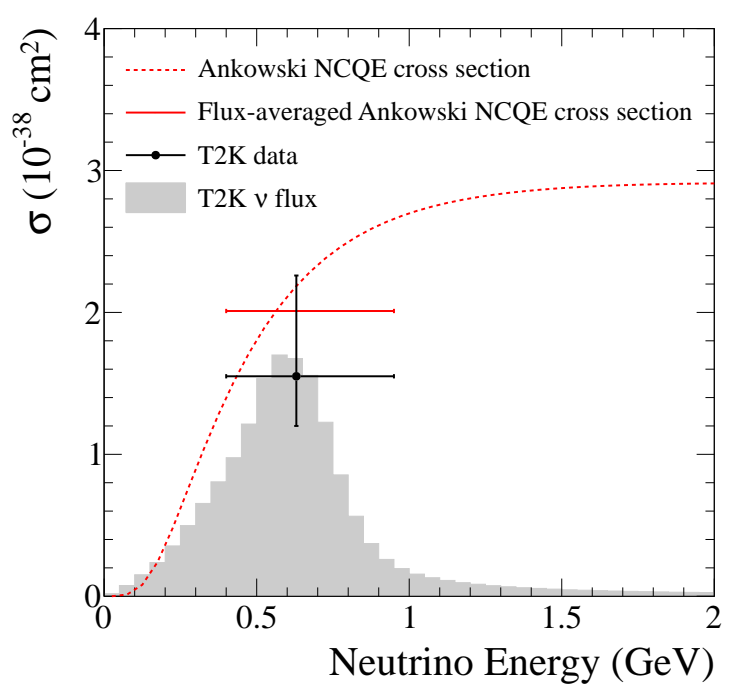

Figure 6: The T2K measurement of the flux-averaged NCQE cross section, represented by a black point. The dashed line shows the predicted cross section versus neutrino energy, the solid horizontal line shows the predicted flux-averaged cross section. The horizontal error bar is placed at the central value from our data and represents $68 \%$ of the flux at each side of the median energy. The solid gray histogram shows the unoscillated $\mathrm{T} 2 \mathrm{~K}$ neutrino flux. 


\section{Summary}

We observed gamma-rays produced in neutral current quasi-elastic interactions in SK using the T2K beam. In this report, $3.01 \times 10^{20}$ protons of target data (T2K Run 1-3) were analyzed. In our analysis, the data reduction resulted in 43 event candidates, which was consistent with the theoretical prediction at the one sigma level. The measured cross section was $1.55 \times 10^{-38} \mathrm{~cm}^{2}$. This is the first measurement of the cross section of neutrino-oxygen NCQE interactions [13].

\section{Acknowledgements}

The author would like to thank the T2K collaboration for this work. This work was partially supported by the JSPS KAKENHI Grant Number 24103004.

\section{References}

[1] K. Langanke, P. Vogel, and E. Kolbe, Phys. Rev. Lett. 76, 2629 (1996).

[2] A. M. Ankowski, O. Benhar, T. Mori, R. Yamaguchi, and M. Sakuda, Phys. Rev. Lett. 108, 052505 (2012).

[3] K. Bays et al. (Super-Kamiokande Collaboration), Phys. Rev. D 85, 052007 (2012).

[4] K. Ueno et al. (Super-Kamiokande Collaboration), Astropart. Phys. 36, 131 (2012).

[5] K. Abe et al. (T2K Collaboration), Phys. Rev. Lett. 112, 061802 (2014).

[6] S. Fukuda et al. (Super-Kamiokande Collaboration), Nucl. Instrum. Meth. A501, 418 (2003).

[7] H. Nishino et al. Nucl. Instrum. Meth. A610, 710 (2009).

[8] K. Abe et al. (Super-Kamiokande Collaboration), Nucl. Instrum. Meth. A737C, 253 (2014).

[9] M. Nakahata et al. (Super-Kamiokande Collaboration), Nucl. Instrum. Meth. A421, 113 (1999).

[10] E. Blaufuss et al. (Super-Kamiokande Collaboration), Nucl. Instrum. Meth. A458, 636 (2001).

[11] K. Kobayashi et al., arXiv:nucl-ex/0604006.

[12] K. Abe et al. (Super-Kamiokande Collaboration), Phys. Rev. D 83, 052010 (2011).

[13] K. Abe et al. (T2K Collaboration), Phys. Rev. D 90, 072012 (2014). 